Bull. Astr. Soc. India (2011) 39, 1,20

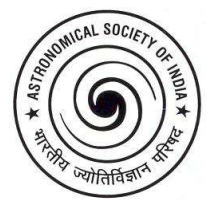

\title{
Key problems in black hole physics today
}

\author{
Pankaj S. Joshi, ${ }^{*}$ \\ Tata Institute of Fundamental Research, Homi Bhabha Road, Mumbai 400005, India
}

Received 2011 April 04; accepted April 14

\begin{abstract}
We review here some of the major open issues and challenges in black hole physics today, and the current progress on the same. It is pointed out that to secure a concrete foundation for the basic theory as well as astrophysical applications for black hole physics, it is essential to gain a suitable insight into these questions. In particular, we discuss the recent results investigating the final fate of a massive star within the framework of the Einstein gravity, and the stability and genericity aspects of the gravitational collapse outcomes in terms of black holes and naked singularities. Recent developments such as spinning up a black hole by throwing matter into it, and physical effects near naked singularities are considered. It is pointed out that some of the new results obtained in recent years in the theory of gravitational collapse imply interesting possibilities and understanding for the theoretical advances in gravity as well as towards new astrophysical applications.
\end{abstract}

Keywords : black hole physics - gravitation

\section{Introduction}

The fundamental question of the final fate of a massive star, when it exhausts its internal nuclear fuel and collapses continually under the force of its own gravity, was highlighted by Chandrasekhar way back in 1934 (Chandrasekhar 1934), who pointed out:

"Finally, it is necessary to emphasize one major result of the whole investigation, namely, that the life-history of a star of small mass must be essentially different from the life-history of a star of large mass. For a star of small mass the natural white-dwarf stage is an initial step towards complete extinction. A star of large mass $\left(>M_{c}\right)$ cannot pass into the white-dwarf stage, and one is left speculating on other possibilities."

\footnotetext{
*e-mail: psj@tifr.res.in
} 
We can see the seeds of modern black hole physics already present in the inquiry made above on the final fate of massive stars. The issue of endstate of large mass stars has, however, remained unresolved and elusive for a long time of many decades after that. In fact, a review of the status of the subject many decades later notes, "Any stellar core with a mass exceeding the upper limit that undergoes gravitational collapse must collapse to indefinitely high central density... to form a (spacetime) singularity" (Report of the Physics Survey Committee 1986).

The reference above is to the prediction by general relativity, that under reasonable physical conditions, the gravitationally collapsing massive star must terminate in a spacetime singularity (Hawking \& Ellis 1973). The densities, spacetime curvatures, and all physical quantities must typically go to arbitrarily large values close to such a singularity. The above theoretical result on the existence of singularities is, however, of a rather general nature, and provides no information on the nature and structure of such singularities. In particular, it gives us no information as to whether such singularities, when they form, will be covered in horizons of gravity and hidden from us, or alternatively these could be visible to external observers in the Universe.

One of the key questions in black hole physics today therefore is, are such singularities resulting from collapse, which are super-ultra-dense regions forming in spacetime, visible to external observers in the Universe? This is one of the most important unresolved issues in gravitation theory currently. Theorists generally believed that in such circumstances, a black hole will always form covering the singularity, which will then be always hidden from external observers. Such a black hole is a region of spacetime from which no light or particles can escape. The assumption that spacetime singularities resulting from collapse would be always covered by black holes is called the Cosmic Censorship Conjecture (CCC; Penrose 1969). As of today, we do not have any proof or any specific mathematical formulation of the CCC available within the framework of gravitation theory.

If the singularities were always covered in horizons and if CCC were true, that would provide a much needed basis for the theory and astrophysical applications of black holes. On the other hand, if the spacetime singularities which result from a continual collapse of a massive star were visible to external observers in the Universe, we would then have the opportunity to observe and investigate the super-ultra-dense regions in the Universe, which form due to gravitational collapse and where extreme high energy physics and also quantum gravity effects will be at work.

My purpose here is to review the above and some of the related key issues in gravitation theory and black hole physics today. This will be of course from a perspective of what I think are the important problems, and no claim to completeness is made. In Section 2, we point out that in view of the lack of any theoretical progress on CCC, the important way to make any progress on this problem is to make a detailed and extensive study of gravitational collapse in general relativity. Some recent progress in this direction is summarized. While we now seem to have a good understanding of the black hole and naked singularity formations as final fate of collapse in many gravitational collapse models, the key point now is to understand the genericity and stability of these outcomes, as viewed in a suitable framework. Section 3 discusses these issues in some detail. Recent developments on throwing matter into a black hole and the effect it may have on its 
horizon are pointed out in Section 4, and certain quantum aspects are also discussed. The issue of predictability or its breakdown in gravitational collapse is discussed in Section 5. We conclude by giving a brief idea of the future outlook and possibilities in the final section.

\section{What is the final fate of a massive star?}

While Chandra's work pointed out the stable configuration limit for the formation of a white dwarf, the issue of the final fate of a star which is much more massive (e.g. tens of solar masses) remains very much open even today. Such a star cannot settle either as a white dwarf or as a neutron star.

The issue is clearly important both in high energy astrophysics and in cosmology. For example, our observations today on the existence of dark energy in the Universe and its acceleration are intimately connected to the the observations of Type Ia supernovae in the Universe. The observational evidence coming from these supernovae, which are exploding stars in the faraway Universe, tells us on how the Universe may be accelerating away and the rate at which such an acceleration is taking place. While Type Ia supernovae result from the explosion of a white dwarf star, at the heart of a Type II supernova underlies the phenomenon of a catastrophic gravitational collapse of a massive star, wherein a powerful shock wave is generated, blowing off the outer layers of the star.

If such a star is able to throw away enough of matter in such an explosion, it might eventually settle as a neutron star. But otherwise, or if further matter is accreted onto the neutron star, there will be a further continual collapse, and we shall have to then explore and investigate the question of the final fate of such a massive collapsing star. But other stars, which are more massive and well above the normal supernova mass limits must straightaway enter a continual collapse mode at the end of their life cycle, without an intermediate neutron star stage. The final fate of the star in this case must be decided by general relativity alone.

The point here is, more massive stars which are tens of times the mass of the Sun burn much faster and are far more luminous. Such stars then cannot survive more than about ten to twenty million years, which is a much shorter life span compared to stars like the Sun, which live billions of years. Therefore, the question of the final fate of such short-lived massive stars is of central importance in astronomy and astrophysics.

What happens then, in terms of the final outcome, when such a massive star dies after exhausting its internal nuclear fuel? As we indicated above, the general theory of relativity predicts that the collapsing massive star must terminate in a spacetime singularity, where the matter energy densities, spacetime curvatures and other physical quantities blow up. It then becomes crucial to know whether such super-ultra-dense regions, forming in stellar collapse, are visible to an external observer in the Universe, or whether they will be always hidden within a black hole and an event horizon that could form as the star collapses. This is one of the most important issues in black hole physics today. 


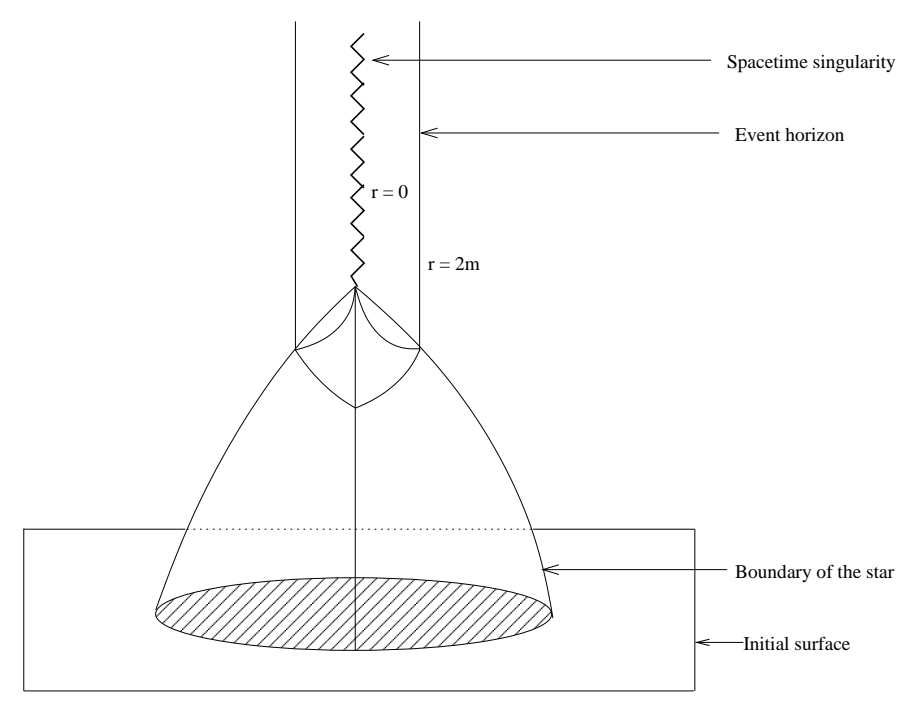

Figure 1. Dynamical evolution of a homogeneous spherical dust cloud collapse, as described by the Oppenheimer-Snyder-Datt solution.

The issue has to be probed necessarily within the framework of a suitable theory of gravity, because the strong gravity effects will be necessarily important in such a scenario. This was done for the first time in the late 1930s, by the works of Oppenheimer and Snyder, and Datt (Oppenheimer \& Snyder 1939; Datt 1938). They used the general theory of relativity to examine the final fate of an idealized massive matter cloud, which was taken to be a spatially homogeneous ball which had no rotation or internal pressure, and was assumed to be spherically symmetric. The dynamical collapse studied here resulted in the formation of a spacetime singularity, which was preceded by the development of an event horizon, which created a black hole in the spacetime. The singularity was hidden inside such a black hole, and the collapse eventually settled into a final state which was the Schwarzschild geometry (see Fig. 1).

There was, however, not much attention paid to this model at that time, and it was widely thought by gravitation theorists as well as astronomers that it would be absurd for a star to reach such a final ultra-dense state of its evolution. It was in fact only as late as 1960s, that a resurgence of interest took place in the topic, when important observational developments in astronomy and astrophysics revealed several very high energy phenomena in the Universe, such as quasars and radio galaxies, where no known physics was able to explain the observations of such extremely high energy phenomena in the cosmos. Attention was drawn then to dynamical gravitational collapse and its final fate, and in fact the term 'black hole' was also popularized just around the same time in 1969, by John Wheeler.

The CCC also came into existence in 1969. It suggested and assumed that what happens in the Oppenheimer-Snyder-Datt (OSD) picture of gravitational collapse, as discussed above, would be the generic final fate of a realistic collapsing massive star in general. In other words, it was 


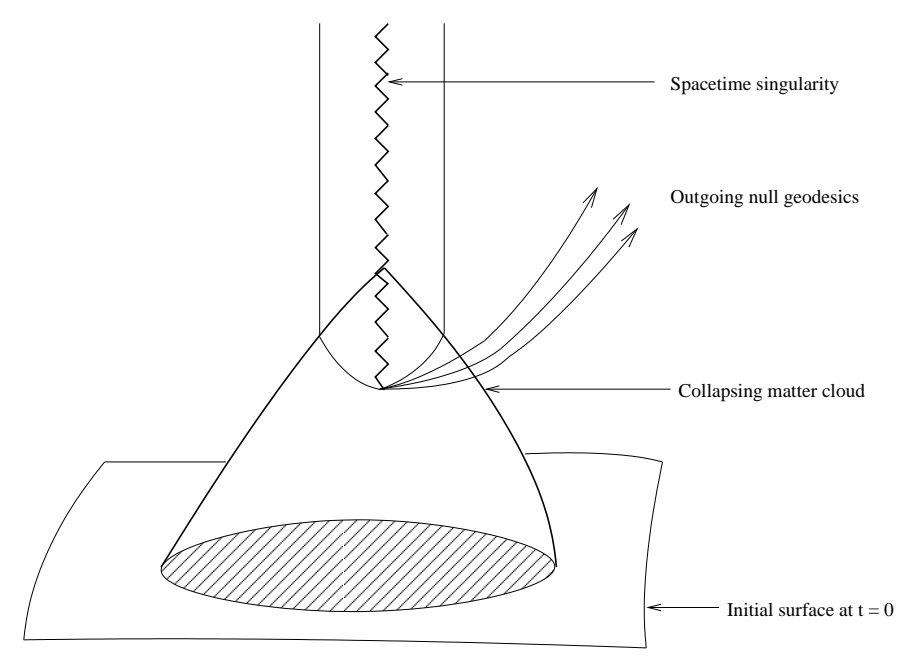

Figure 2. A spacetime singularity of gravitational collapse which is visible to external observers in the Universe, in violation to the cosmic censorship conjecture.

assumed that the collapse of a realistic massive star will terminate in a black hole, which hides the singularity, and thus no visible or naked singularities will develop in gravitational collapse. Many important developments then took place in black hole physics which started in earnest, and several important theoretical aspects as well as astrophysical applications of black holes started developing. The classical as well as quantum aspects of black holes were then explored and interesting thermodynamic analogies for black holes were also developed. Many astrophysical applications for the real Universe then started developing for black holes, such as making models using black holes for phenomena such as jets from the centres of galaxies and the extremely energetic gamma rays bursts.

The key issue raised by the CCC, however, still remained very much open, namely whether a real star will necessarily go the OSD way for its final state of collapse, and whether the final singularity will be always necessarily covered within an event horizon. This is because real stars are inhomogeneous, have internal pressure forces and so on, as opposed to the idealized OSD assumptions. This remains an unanswered question, which is one of the most important issues in gravitation physics and black hole physics today. A spacetime singularity that is visible to faraway observers in the Universe is called a naked singularity (see Fig. 2). The point here is, while general relativity predicts the existence of singularity as the endstate for collapse, it gives no information at all on the nature or structure of such singularities, and whether they will be covered by event horizons, or would be visible to external observers in the Universe.

There is no proof, or even any mathematically rigorous statement available for CCC after many decades of serious effort. What is really needed to resolve the issue is gravitational collapse models for a realistic collapse configuration, with inhomogeneities and pressures included. The effects need to be worked out and studied in detail within the framework of Einstein gravity. Only 
such considerations will allow us to determine the final fate of collapse in terms of either a black hole or a naked singularity final state.

Over the past couple of decades, many such collapse models have been worked out and studied in detail. The generic conclusion that has emerged out of these studies is that both the black holes and naked singularity final states do develop as collapse endstates, for a realistic gravitational collapse that incorporates inhomogeneities as well as non-zero pressures within the interior of the collapsing matter cloud. Subject to various regularity and energy conditions to ensure the physical reasonableness of the model, it is the initial data, in terms of the initial density, pressures, and velocity profiles for the collapsing shells, that determine the final fate of collapse as either a naked singularity or a black hole (for further detail and references see e.g. Joshi 2008).

\section{The genericity and stability of collapse outcomes}

While general relativity may predict the existence of both black holes and naked singularities as collapse outcome, an important question then is, how would a realistic continual gravitational collapse of a massive star in nature would end up. Thus the key issue under active debate now is the following: Even if naked singularities did develop as collapse end states, would they be generic or stable in some suitably well-defined sense, as permitted by the gravitation theory? The point here is, if naked singularity formation in collapse was necessarily 'non-generic' in some appropriately well-defined sense, then for all practical purposes, a realistic physical collapse in nature might always end up in a black hole, whenever a massive star ended its life.

In fact, such a genericity requirement has been always discussed and desired for any possible mathematical formulation for CCC right from its inception. However, the main difficulty here has again been that, there is no well-defined or precise notion of genericity available in gravitation theory and the general theory of relativity. Again, it is only various gravitational collapse studies that can provide us with more insight into this genericity aspect also.

A result that is relevant here is the following (Joshi \& Dwivedi, 1999; Goswami \& Joshi, 2007). For a spherical gravitational collapse of a rather general (type I) matter field, satisfying the energy and regularity conditions, given any regular density and pressure profiles at the initial epoch, there always exist classes of velocity profiles for the collapsing shells and dynamical evolutions as determined by the Einstein equations, that, depending on the choice made, take the collapse to either a black hole or naked singularity final state (see e.g. Fig. 3 for a schematic illustration of such a scenario).

Such a distribution of final states of collapse in terms of the black holes and naked singularities can be seen much more transparently when we consider a general inhomogeneous dust collapse, for example, as discussed by Mena, Tavakol \& Joshi (2000) (see Fig.4).

What determines fully the final fate of collapse here are the initial density and velocity profiles for the collapsing shells of matter. One can see here clearly how the different choices of 


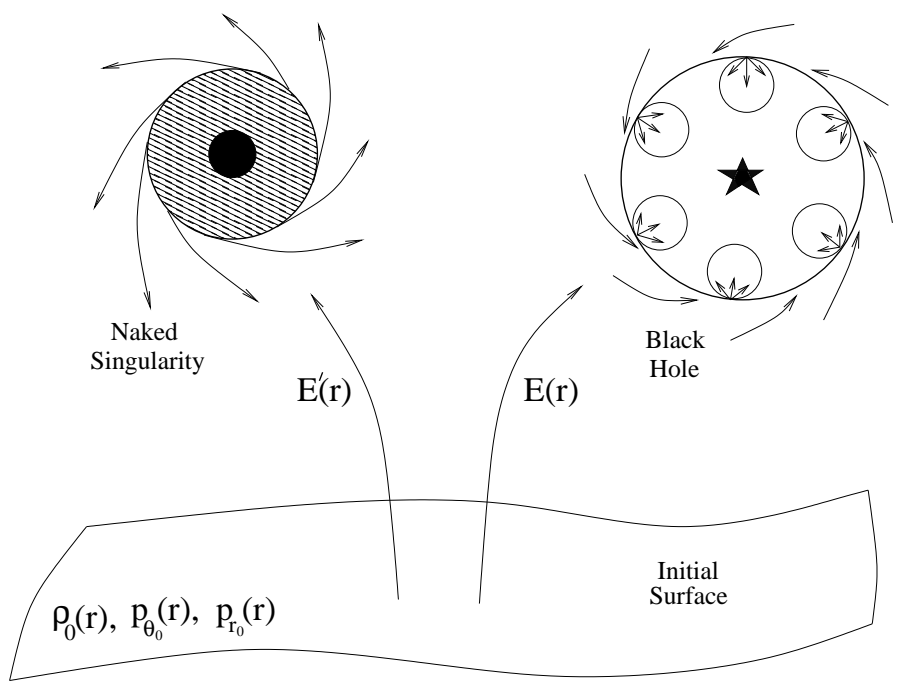

Figure 3. Evolution of spherical collapse for a general matter field with inhomogeneities and non-zero pressures included.

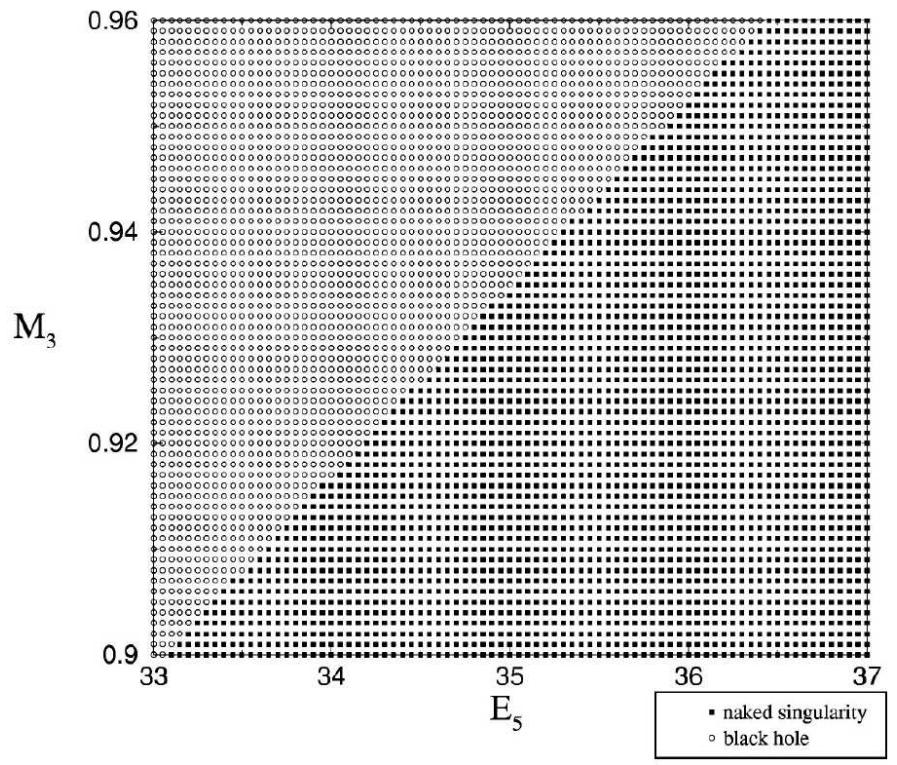

Figure 4. Collapse final states for inhomogeneous dust in terms of initial mass and velocity profiles for the collapsing shells. 
these profiles for the collapsing cloud distinguish between the two final states of collapse, and how each of the black hole and naked singularity states appears to be 'generic' in terms of their being distributed in the space of final states. Typically, the result we have here is, given any regular initial density profile for the collapsing dust cloud, there are velocity profiles that take the collapse to a black hole final state, and there are other velocity profiles that take it to naked singularity final state. In other words, the overall available velocity profiles are divided into two distinct classes, namely the ones which take the given density profile into black holes, and the other ones that take the collapse evolution to a naked singularity final state. The same holds conversely also, namely if we choose a specific velocity profile, then the overall density profile space is divided into two segments, one taking the collapse to black hole final states and the other taking it to naked singularity final states. The clarity of results here gives us much understanding on the final fate of a collapsing matter cloud.

Typically, all stars have a higher density at the centre, which slowly decreases as one moves away. So it is very useful to incorporate inhomogeneity into dynamical collapse considerations. However, much more interesting is the collapse with non-zero pressures which are very important physical forces within a collapsing star. We briefly consider below a typical scenario of collapse with a non-zero pressure component, and for further details we refer to Joshi \& Malafarina (2011).

For a possible insight into genericity of naked singularity formation in collapse, we investigated the effect of introducing small tangential pressure perturbations in the collapse dynamics of the classic Oppenheimer-Snyder-Datt gravitational collapse, which is an idealized model assuming zero pressure, and which terminates in a black hole final fate as discussed above. Thus we study the stability of the OSD black hole under introduction of small tangential stresses.

It is seen explicitly that there exist classes of stress perturbations such that the introduction of a smallest tangential pressure within the collapsing OSD cloud changes the endstate of collapse to formation of a naked singularity, rather than a black hole. What follows is that small stress perturbations within the collapsing cloud change the final fate of the collapse from being a black hole to a naked singularity. This can also be viewed as perturbing the spacetime metric of the cloud in a small way. Thus we can understand here the role played by tangential pressures in a well-known gravitational collapse scenario. A specific and physically reasonable but generic enough class of perturbations is considered so as to provide a good insight into the genericity of naked singularity formation in collapse when the OSD collapse model is perturbed by introduction of a small pressure. Thus we have an important insight into the structure of the censorship principle which as yet remains to be properly understood.

The general spherically symmetric metric describing the collapsing matter cloud can be written as,

$$
d s^{2}=-e^{2 \sigma(t, r)} d t^{2}+e^{2 \psi(t, r)} d r^{2}+R(t, r)^{2} d \Omega^{2}
$$

with the stress-energy tensor for a generic matter source being given by, $T_{t}^{t}=-\rho, T_{r}^{r}=p_{r}, T_{\theta}^{\theta}=$ $T_{\phi}^{\phi}=p_{\theta}$. The above is a general scenario, in that it involves no assumptions about the form of the matter or the equation of state. 
As a step towards deciding the stability or otherwise of the OSD collapse model under the injection of small tangential stress perturbations, we consider the dynamical development of the collapsing cloud, as governed by the Einstein equations. The visibility or otherwise of the final singularity that develops in collapse is determined by the behaviour of the apparent horizon in the spacetime, which is the boundary of the trapped surface region that develops as the collapse progresses. First, we define a scaling function $v(r, t)$ by the relation $R=r v$. The Einstein equations for the above spacetime geometry can then be written as,

$$
\begin{aligned}
p_{r} & =-\frac{\dot{F}}{R^{2} \dot{R}}, \rho=\frac{F^{\prime}}{R^{2} R^{\prime}}, \\
\sigma^{\prime} & =2 \frac{p_{\theta}-p_{r}}{\rho+p_{r}} \frac{R^{\prime}}{R}-\frac{p_{r}^{\prime}}{\rho+p_{r}}, \\
2 \dot{R}^{\prime} & =R^{\prime} \frac{\dot{G}}{G}+\dot{R} \frac{H^{\prime}}{H}, \\
F & =R(1-G+H),
\end{aligned}
$$

The functions $H$ and $G$ in the above are defined as, $H=e^{-2 \sigma(r, v)} \dot{R}^{2}, G=e^{-2 \psi(r, v)} R^{\prime 2}$. The above are five equations in seven unknowns, namely $\rho, p_{r}, p_{\theta}, R, F, G, H$. Here $\rho$ is the mass-energy density, $p_{r}$ and $p_{\theta}$ are the radial and tangential stresses respectively, $R$ is the physical radius for the matter cloud, and $F$ is the Misner-Sharp mass function.

It is possible now, with the above definitions of $v, H$ and $G$, to substitute the unknowns $R, H$ with $v, \sigma$. Then, without loss of generality, the scaling function $v$ can be written as $v\left(t_{i}, r\right)=1$ at the initial time $t_{i}=0$, when the collapse begins. It then goes to zero at the spacetime singularity $t_{s}$, which corresponds to $R=0$, and thus we have $v\left(t_{s}, r\right)=0$. This amounts to the scaling $R=r$ at the initial epoch of the collapse, which is an allowed freedom. The collapse condition here is $\dot{R}<0$ throughout the evolution, and this is equivalent to $\dot{v}<0$.

One can integrate the Einstein equations, at least up to one order, to reduce them to a first order system, to obtain the function $v(t, r)$. This function, which is monotonically decreasing in $t$ can be inverted to obtain the time needed by a matter shell at any radial value $r$ to reach the event with a particular value $v$. We can then write the function $t(r, v)$ as,

$$
t(r, v)=\int_{v}^{1} \frac{e^{-\sigma}}{\sqrt{\frac{F}{r^{3} \tilde{v}}+\frac{b e^{2 r A}-1}{r^{2}}}} d \tilde{v} .
$$

The function $A(r, v)$ in the above depends on the nature of the tangential stress perturbations chosen. The time taken by the shell at $r$ to reach the spacetime singularity at $v=0$ is then $t_{s}(r)=t(r, 0)$.

Since $t(r, v)$ is in general at least $C^{2}$ everywhere in the spacetime (because of the regularity of the functions involved), and is continuous at the centre, we can write it as,

$$
t(r, v)=t(0, v)+r \chi(v)+O\left(r^{2}\right)
$$


Then, by continuity, the time for a shell located at any $r$ close to the centre to reach the singularity is given as,

$$
t_{s}(r)=t_{s}(0)+r \chi(0)+O\left(r^{2}\right)
$$

Basically, this means that the singularity curve should have a well-defined tangent at the center. Regularity at the center also implies that the metric function $\sigma$ cannot have constant or linear terms in $r$ in a close neighborhood of $r=0$, and it must go as $\sigma \sim r^{2}$ near the center. Therefore the most general choice of the free function $\sigma$ is,

$$
\sigma(r, v)=r^{2} g(r, v)
$$

Since $g(r, v)$ is a regular function (at least $C^{2}$ ), it can be written near $r=0$ as,

$$
g(r, v)=g_{0}(v)+g_{1}(v) r+g_{2}(v) r^{2}+\ldots
$$

We can now investigate how the OSD gravitational collapse scenario, which gives rise to a black hole as the final state, gets altered when small stress perturbations are introduced in the dynamical evolution of collapse. For that we first note that the dust model is obtained if $p_{r}=p_{\theta}=0$ in the above. In that case, $\sigma^{\prime}=0$ and together with the condition $\sigma(0)=0$ gives $\sigma=0$ identically. In the OSD homogeneous collapse to a black hole, the trapped surfaces and the apparent horizon develop much earlier before the formation of the final singularity. But when density inhomogeneities are allowed in the initial density profile, such as a higher density at the centre of the star, then the trapped surface formation is delayed in a natural manner within the collapsing cloud. Then the final singularity becomes visible to faraway observers in the Universe (e.g. Joshi, Dadhich \& Maartens 2002).

The OSD case is obtained from the inhomogeneous dust case, when we assume further that the collapsing dust is necessarily homogeneous at all epochs of collapse. This is of course an idealized scenario because realistic stars would have typically higher densities at the centre, and they also would have non-zero internal stresses. The conditions that must be imposed to obtain the OSD case from the above are given by $M=M_{0} v=v(t) b_{0}(r)=k$. Then we have $F^{\prime}=3 M_{0} r^{2}$, $R^{\prime}=v$, the energy density is homogeneous throughout the collapse, and the density is given by $\rho=\rho(t)=3 M_{0} / v^{3}$. The spacetime geometry then becomes the Oppenheimer-Snyder metric, which is given by,

$$
d s^{2}=-d t^{2}+\frac{v^{2}}{1+k r^{2}} d r^{2}+r^{2} v^{2} d \Omega^{2},
$$

where the function $v(t)$ is a solution of the equation of motion, $\frac{d v}{d t}=\sqrt{\left(M_{0} / v\right)+k}$, obtained from the Einstein equation. In this case we get $\chi(0)=0$ identically. All the matter shells then collapse into a simultaneous singularity, which is necessarily covered by the event horizon that developed in the spacetime at an earlier time. Therefore the final fate of collapse is a black hole.

To explore the effect of introducing small pressure perturbations in the above OSD scenario and to study the models thus obtained which are close to the Oppenheimer-Snyder, we can relax one or more of the above conditions. If the collapse outcome is not a black hole, the final collapse 
to singularity cannot be simultaneous. We can thus relax the condition $v=v(t)$ above, allowing for $v=v(t, r)$. We keep the other conditions of the OSD model unchanged, so as not to depart too much from the OSD model, and this should bring out more clearly the role played by the stress perturbations in the model. We know that the metric function $\sigma(t, r)$ must identically vanish for the dust case. On the other hand, the above amounts to allowing for small perturbations in $\sigma$, and allowing it to be non-zero now. This is equivalent to introducing small stress perturbations in the model, and it is seen that this affects the apparent horizon developing in the collapsing cloud. We note that taking $M=M_{0}$ leads to $F=r^{3} M_{0}$.

In this case, in the small $r$ limit we obtain $G(r, t)=b(r) e^{2 \sigma(r, v)}$. The radial stress $p_{r}$ vanishes here as $\dot{F}=0$, and the tangential pressure turns out to have the form, $p_{\theta}=p_{1} r^{2}+p_{2} r^{3}+\ldots$, where $p_{1}, p_{2}$ are evaluated in terms of coefficients of $m, g$, and $R$ and its derivatives, and we get,

$$
p_{\theta}=3 \frac{M_{0} g_{0}}{v R^{\prime 2}} r^{2}+\frac{9}{2} \frac{M_{0} g_{1}}{v R^{\prime 2}} r^{3}+\ldots
$$

As seen above, the choice of the sign of the functions $g_{0}$ and $g_{1}$ is enough to ensure positivity or negativity of the pressure $p_{\theta}$. The first order coefficient $\chi$ in the equation of the time curve of the singularity $t_{s}(r)$ is now obtained as,

$$
\chi(0)=-\int_{0}^{1} \frac{v^{\frac{3}{2}} g_{1}(v)}{\left(M_{0}+v k+2 v g_{0}(v)\right)^{\frac{3}{2}}} d v .
$$

As we have noted above, it is the quantity $\chi(0)$ that governs the nature of the singularity curve, and whether it is increasing or decreasing away from the center. It can be seen from above that it is the matter initial data in terms of the density and stress profiles, the velocity of the collapsing shells, and the allowed dynamical evolutions that govern and fix the value of $\chi(0)$.

The apparent horizon in the spacetime and the trapped surface formation as the collapse evolves is also governed by the quantity $\chi(0)$, which in turn governs the nakedness or otherwise of the singularity. The equation for the apparent horizon is given by $F / R=1$. This is analogous to that of the dust case since $F / R=r M / v$ in both these cases. So the apparent horizon curve $r_{a h}(t)$ is given by

$$
r_{a h}^{2}=\frac{v_{a h}}{M_{0}},
$$

with $v_{a h}=v\left(r_{a h}(t), t\right)$, which can also be inverted as a time curve for the apparent horizon $t_{a h}(r)$. The visibility of the singularity at the center of the collapsing cloud to faraway observers is determined by the nature of this apparent horizon curve which is given by,

$$
t_{a h}(r)=t_{s}(r)-\int_{0}^{v_{a h}} \frac{e^{-\sigma}}{\sqrt{\frac{M_{0}}{v}+\frac{b e^{2 \sigma}-1}{r^{2}}}} d v
$$

where the $t_{s}(r)$ is the singularity time curve, and its initial point is $t_{0}=t_{s}(0)$. Near $r=0$ we then get,

$$
t_{a h}(r)=t_{0}+\chi(0) r+o\left(r^{2}\right)
$$


From these considerations, it is possible to see how the stress perturbations affect the time of formation of the apparent horizon, and therefore the formation of a black hole or naked singularity. A naked singularity would typically occur as a collapse endstate when a comoving observer at a fixed $r$ value does not encounter any trapped surfaces before the time of singularity formation. For a black hole to form, trapped surfaces must develop before the singularity. Therefore it is required that,

$$
t_{a h}(r) \leq t_{0} \text { for } r>0 \text {, near } r=0 .
$$

As can be seen from above, for all functions $g_{1}(v)$ for which $\chi(0)$ is positive, this condition is violated and in that case the apparent horizon is forced to appear after the formation of the central singularity. In that case, the apparent horizon curve begins at the central singularity $r=0$ at $t=t_{0}$ and increases with increasing $r$, moving to the future. Then we have $t_{a h}>t_{0}$ for $r>0$ near the center. The behaviour of outgoing families of null geodesics has been analyzed in detail in the case when $\chi(0)>0$ and we can see that the geodesics terminate at the singularity in the past. Thus timelike and null geodesics come out from the singularity, making it visible to external observers (Joshi \& Dwivedi 1999).

One thus sees that it is the term $g_{1}$ in the stresses $p_{\theta}$ which decides either the black hole or naked singularity as the final fate for the collapse. We can choose it to be arbitrarily small, and it is then possible to see how introducing a generic tangential stress perturbation in the model would change drastically the final outcome of the collapse. For example, for all non-vanishing tangential stresses with $g_{0}=0$ and $g_{1}<0$, even the slightest perturbation of the OppenheimerSnyder-Datt scenario, injecting a small tangential stress would result in a naked singularity. The space of all functions $g_{1}$ that make $\chi(0)$ positive, which includes all the strictly negative functions $g_{1}$, causes the collapse to end in a naked singularity. While this is an explicit example, it is by no means the only class. The important feature of this class is that it corresponds to a collapse model for a simple and straightforward perturbation of the Oppenheimer-Snyder-Datt spacetime metric.

In this case, the geometry near the centre can be written as,

$$
d s^{2}=-\left(1-2 g_{1} r^{3}\right) d t^{2}+\frac{\left(v+r v^{\prime}\right)^{2}}{1+k r^{2}-2 g_{1} r^{3}} d r^{2}+r^{2} v^{2} d \Omega^{2},
$$

The metric above satisfies the Einstein equations in the neighborhood of the center of the cloud when the function $g_{1}(v)$ is small and bounded. We can take $0<\left|g_{1}(v)\right|<\epsilon$, so that, the smaller we take the parameter $\epsilon$, the bigger will be the radius where the approximation is valid. We can consider here the requirement that a realistic matter model should satisfy some energy conditions ensuring the positivity of mass and energy density. The weak energy condition would imply restrictions on the density and pressure profiles. The energy density as given by the Einstein equation must be positive. Since $R$ is positive, to ensure positivity of $\rho$ we require $F>0$ and $R^{\prime}>0$. The choice of positive $M(r)$, which clearly holds for $M_{0}>0$, and is physically reasonable, ensures positivity of the mass function. Here $R^{\prime}>0$ is a sufficient condition for the avoidance of shell crossing singularities. The tangential stress can now be written, with $p_{r}=0$, and is given by

$$
p_{\theta}=\frac{1}{2} \frac{R}{R^{\prime}} \rho \sigma^{\prime}
$$


So the sign of the function $\sigma^{\prime}$ would determine the sign of $p_{\theta}$. Positivity of $\rho+p_{\theta}$ is then ensured for small values of $r$ throughout collapse for any form of $p_{\theta}$. In fact, regardless of the values taken by $M$ and $g$, there will always be a neighbourhood of the center $r=0$ for which $\left|p_{\theta}\right|<\rho$ and therefore $\rho+p_{\theta} \geq 0$.

What we see here is that, in the space of initial data in terms of the initial matter densities and velocity profiles, any arbitrarily small neighborhood of the OSD collapse model, which is going to end as a black hole, contains collapse evolutions that go to naked singularities. Such an existence of subspaces of collapse solutions, that go to a naked singularity rather than a black hole, in the arbitrary vicinity of the OSD black hole, presents an intriguing situation. It gives an idea of the richness of structure present in the gravitation theory, and indicates the complex solution space of the Einstein equations which are a complicated set of highly non-linear partial differential equations. What we see here is the existence of classes of stress perturbations such that an arbitrarily small change from the OSD model is a solution going to a naked singularity.

This then provides an intriguing insight into the nature of cosmic censorship, namely that the collapse must necessarily be properly fine-tuned if it is to produce a black hole only as the final endstate. Traditionally it was believed that the presence of stresses or pressures in the collapsing matter cloud would increase the chance of black hole formation, thereby ruling out dust models that were found to lead to a naked singularity as the collapse endstate. It now becomes clear that this is actually not the case. The model described here not only provides a new class of collapses ending in naked singularities, but more importantly, shows how the bifurcation line that separates the phase space of 'black hole formation' from that of 'naked singularity formation' runs directly over the simplest and most studied of black hole scenarios such as the OSD model.

It has to be noted of course that the general issue of stability and genericity of collapse outcomes has been a deep problem in gravitation theory, and requires mathematical breakthroughs as well as evolving further physical understanding of the collapse phenomenon. As noted above, this is again basically connected with the main difficulty of cosmic censorship itself, which is the issue of how to define censorship. However, it is also clear from the discussion above, that consideration of various collapse models along the lines as discussed here does yield considerable insight and inputs in understanding gravitational collapse and its final outcomes.

\section{Spinning up a black hole and quantum aspects}

It is clear that the black hole and naked singularity outcomes of a complete gravitational collapse for a massive star are very different from each other physically, and would have quite different observational signatures. In the naked singularity case, if it occurs in nature, we have the possibility of observing the physical effects happening in the vicinity of the ultra-dense regions that form in the very final stages of collapse. However, in a black hole scenario, such regions are necessarily hidden within the event horizon of gravity. The fact that a slightest stress perturbation of the OSD collapse could change the collapse final outcome drastically, as we noted in the previous section, changing it from black hole formation to a naked singularity, means that the naked singularity fi- 


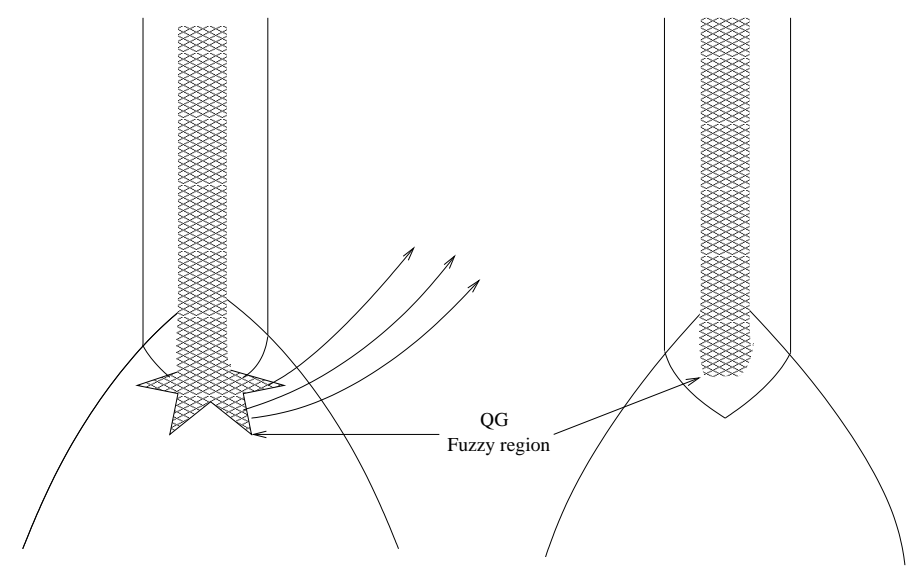

Figure 5. Even if the naked singularity is resolved by the quantum gravity effects, the ultra-strong gravity region that developed in gravitational collapse will still be visible to external observers in the Universe.

nal state for a collapsing star must be studied very carefully to deduce its physical consequences, which are not well understood so far.

It is, however, widely believed that when we have a reasonable and complete quantum theory of gravity available, all spacetime singularities, whether naked or those hidden inside black holes, will be resolved away. As of now, it remains an open question if quantum gravity will remove naked singularities. After all, the occurrence of spacetime singularities could be a purely classical phenomenon, and whether they are naked or covered should not be relevant, because quantum gravity will possibly remove them all any way. But one may argue that looking at the problem this way is missing the real issue. It is possible that in a suitable quantum gravity theory the singularities will be smeared out, though this has not been realized so far. Also there are indications that in quantum gravity also the singularities may not after all go away.

In any case, the important and real issue is, whether the extreme strong gravity regions formed due to gravitational collapse are visible to faraway observers or not. It is quite clear that gravitational collapse would certainly proceed classically, at least till quantum gravity starts governing and dominating the dynamical evolution at scales of the order of the Planck length, i.e. till extreme gravity configurations have been already developed due to collapse. The key point is the visibility or otherwise of such ultra-dense regions whether they are classical or quantum (see Fig. 5).

What is important is, classical gravity implies necessarily the existence of ultra-strong gravity regions, where both classical and quantum gravity come into their own. In fact, if naked singularities do develop in gravitational collapse, then in a literal sense we come face-to-face with the laws of quantum gravity, whenever such an event occurs in the Universe (Wald 1997).

In this way, the gravitational collapse phenomenon has the potential to provide us with a pos- 
sibility of actually testing the laws of quantum gravity. In the case of a black hole developing in the collapse of a finite sized object such as a massive star, such strong gravity regions are necessarily hidden behind an event horizon of gravity, and this would happen well before the physical conditions became extreme near the spacetime singularity. In that case, the quantum effects, even if they caused qualitative changes closer to singularity, will be of no physical consequence. This is because no causal communications are then allowed from such regions. On the other hand, if the causal structure were that of a naked singularity, then communications from such a quantum gravity dominated extreme curvature ball would be visible in principle. This will be so either through direct physical processes near a strong curvature naked singularity, or via the secondary effects, such as the shocks produced in the surrounding medium.

Independently of such possibilities connected with gravitational collapse as above, let us suppose that the collapse terminated in a black hole. It is generally believed that such a black hole will be described by the Kerr metric. A black hole, however, by its very nature accretes matter from the surrounding medium or from a companion star. In that case, it is worth noting here that there has been an active debate in recent years about whether a black hole can survive as it is, when it accretes charge and angular momentum from the surrounding medium.

The point is, there is a constraint in this case for the horizon to remain undisturbed, namely that the black hole must not contain too much of charge and it should not spin too fast. Otherwise, the horizon cannot be sustained. It will breakdown and the singularity within will become visible. The black hole may have formed with small enough charge and angular momentum to begin with; however, we have the key astrophysical process of accretion from its surroundings, of the debris and outer layers of the collapsing star. This matter around the black hole will fall into the same with great velocity, which could be classical or quantized, and with either charge or angular momentum or perhaps both. Such in-falling particles could 'charge-up' or 'over-spin' the black hole, thus eliminating the event horizon. Thus, the very fundamental characteristic of a black hole, namely its trait of gobbling up the matter all around it and continuing to grow becomes its own nemesis and a cause of its own destruction.

Thus, even if a massive star collapsed into a black hole rather than a naked singularity, important issues remain such as the stability against accretion of particles with charge or large angular momentum, and whether that can convert the black hole into a naked singularity by eliminating its event horizon. Many researchers have claimed this is possible, and have given models to create naked singularities this way. But there are others who claim there are physical effects which would save the black hole from over-spinning this way and destroying itself, and the issue is very much open as yet. The point is, in general, the stability of the event horizon and the black hole continues to be an important issue for black holes that developed in gravitational collapse. For a recent discussion on some of these issues, we refer to Matsas \& da Silva (2007), Matsas et al. (2009), Hubeny (1999), Hod (2008), Richartz \& Saa (2008), Jacobson \& Sotiriou (2009, 2010a,b), Barausse, Cardoso \& Khanna (2010), and references therein.

The primary concern of the cosmic censorship hypothesis is the formation of black holes as collapse endstates. Their stability, as discussed above, is only a secondary issue. Therefore, what 


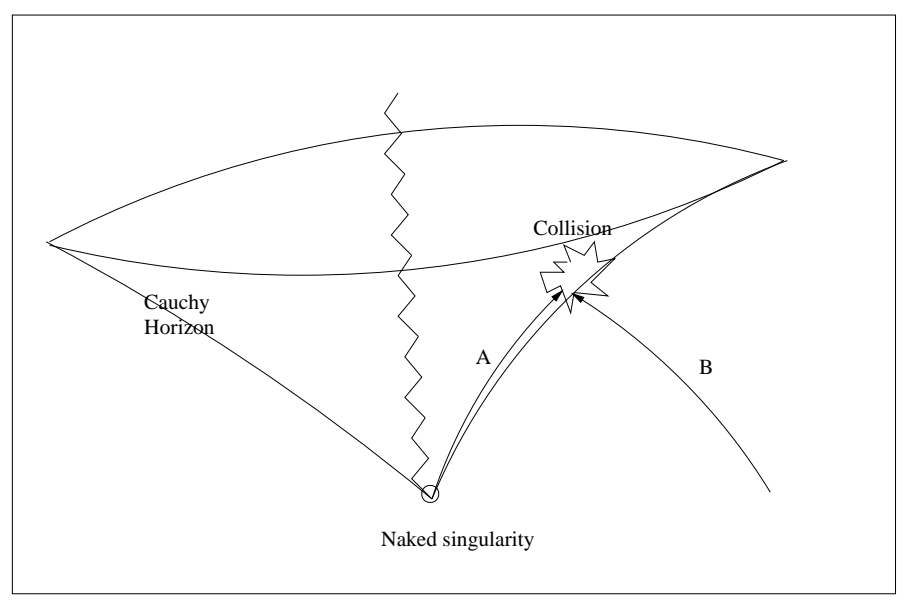

Figure 6. The existence of a naked singularity is typically characterized by existence of a Cauchy horizon in the spacetime. Very high energy particle collisions can occur close to such a Cauchy horizon.

this means for cosmic censorship is that the collapsing massive star should not retain or carry too much charge or spin; otherwise it could necessarily end up as a naked singularity, rather than a black hole.

\section{Predictability, Cauchy horizons and all that}

A concern sometimes expressed is that if naked singularities occurred as the final fate of gravitational collapse, that would break the predictability in the spacetime. A naked singularity is characterized by the existence of light rays and particles that emerge from the same. Typically, in all the collapse models discussed above, there is a family of future directed non-spacelike curves that reach external observers, and when extended in the past these meet the singularity. The first light ray that comes out from the singularity marks the boundary of the region that can be predicted from a regular initial Cauchy surface in the spacetime, and that is called the Cauchy horizon for the spacetime. The causal structure of the spacetime would differ significantly in the two cases, when there is a Cauchy horizon and when there is none. A typical gravitational collapse to a naked singularity, with the Cauchy horizon forming is shown in Fig. 6.

The point here is, given a regular initial data on a spacelike hypersurface, one would like to predict the future and past evolutions in the spacetime for all times (see for example, Hawking $\&$ Ellis (1973) for a discussion). Such a requirement is termed the global hyperbolicity of the spacetime. A globally hyperbolic spacetime is a fully predictable universe. It admits a Cauchy surface, the data on which can be evolved for all times in the past as well as in future. Simple enough spacetimes such as the Minkowski or Schwarzschild are globally hyperbolic, but the Reissner-Nordstrom or Kerr geometries are not globally hyperbolic. For further details on these issues, we refer to Hawking \& Ellis (1973) or Joshi (2008). 
Here we would like to mention certain recent intriguing results in connection to the existence of a Cauchy horizon in a spacetime when there is a naked singularity resulting as final fate of a collapse. Let us suppose the collapse resulted in a naked singularity. In that case, there are classes of models where there will be an outflow of energy and radiations of high velocity particles close to the Cauchy horizon, which is a null hypersurface in the spacetime. Such particles, when they collide with incoming particles, would give rise to a very high center of mass energy of collisions. The closer we are to the Cauchy horizon, higher is the center of mass energy of collisions. In the limit of approach to the Cauchy horizon, these energies approach arbitrarily high values and could reach the Planck scale energies (see for example, Patil \& Joshi 2010, 2011a,b; Patil, Joshi \& Malafarina 2011).

It has been observed recently that in the vicinity of the event horizon for an extreme Kerr black hole, if the test particles arrive with fine-tuned velocities, they could undergo very high energy collisions with other incoming particles. In that case, the possibility arises that one could see Planck scale physics or ultra-high energy physics effects near the event horizon, given suitable circumstances (Banados, Silk \& West 2009; Berti et al. 2009; Jacobson \& Sotiriou 2010a,b; Wei et al. 2010; Grib \& Pavlov 2010; Zaslavskii, 2010).

What we mentioned above related to the particle collisions near Cauchy horizon is similar to the situation where the background geometry is that of a naked singularity. These results could mean that in strong gravity regimes, such as those of black holes or naked singularities developing in gravitational collapse, there may be a possibility to observe ultra-high energy physics effects, which would be very difficult to see in the near future in terrestrial laboratories.

While these phenomena give rise to the prospect of observing Planck scale physics near the Cauchy horizon in the gravitational collapse framework, they also raise the following intriguing question. If extreme high energy collisions do take place very close to the null surface which is the Cauchy horizon, then in a certain sense it is essentially equivalent to creating a singularity at the Cauchy horizon. In that case, all or at least some of the Cauchy horizon would be converted into a spacetime singularity, and would effectively mark the end of the spacetime itself. In this case, the spacetime manifold terminates at the Cauchy horizon, whenever a naked singularity is created in gravitational collapse. Since the Cauchy horizon marks in this case the boundary of the spacetime itself, predictability is then restored for the spacetime, because the rest of the spacetime below and in the past of the horizon was predictable before the Cauchy horizon formed.

\section{Future perspectives}

We have pointed out in the considerations here that the final fate of a massive star continues to be a rather exciting research frontier in black hole physics and gravitation theory today. The outcomes here will be fundamentally important for the basic theory and astrophysical applications of black hole physics, and for modern gravitation physics. We highlighted certain key challenges in the field, and also several recent interesting developments were reviewed. Of course, the issues and 
the list given here are by no means complete or exhaustive in any manner, and there are several other interesting problems in the field as well.

In closing, as a summary, we would like to mention here a few points which we think require the most immediate attention, and which will have possibly maximum impact on future development in the field.

1. The genericity of the collapse outcomes, for black holes and naked singularities need to be understood very carefully and in further detail. It is by and large well-accepted now, that the general theory of relativity does allow and gives rise to both black holes and naked singularities as the final outcome of continual gravitational collapse, evolving from a regular initial data, and under reasonable physical conditions. What is not fully clear yet is the distribution of these outcomes in the space of all allowed outcomes of collapse. The collapse models discussed above and considerations we gave here would be of some help in this direction, and may throw some light on the distribution of black holes and naked singularity solutions in the initial data space.

2. Many of the models of gravitational collapse analyzed so far are mainly of spherical symmetric collapse. Therefore, the non-spherical collapse needs to be understood in a much better manner. While there are some models which illustrate what the departures from spherical symmetry could do (see e.g. Joshi \& Krolak 1996), on the whole, not very much is known for non-spherical collapse. Probably numerical relativity could be of help in this direction (see for example Baiotti \& Rezzolla 2006), for a discussion on the evolving developments as related to applications of numerical methods to gravitational collapse issues). Also, another alternative would be to use global methods to deal with the spacetime geometry involved, as used in the case of singularity theorems in general relativity.

3. At the very least, the collapse models studied so far do help us gain much insight into the structure of the cosmic censorship, whatever final form it may have. But on the other hand, there have also been attempts where researchers have explored the physical applications and implications of the naked singularities investigated so far (see e.g. Harada, Iguchi \& Nakao 2000, 2002; Harada et al. (2001) and also references therein).

If we could find astrophysical applications of the models that predict naked singularities, and possibly try to test the same through observational methods and the signatures predicted, that could offer a very interesting avenue to get further insights into this problem as a whole.

4. An attractive recent possibility in that regard is to explore the naked singularities as possible particle accelerators as we pointed out above.

Also, the accretion discs around a naked singularity, wherein the matter particles are attracted towards or repulsed away from the singularities with great velocities could provide an excellent venue to test such effects and may lead to predictions of important observational signatures to distinguish the black holes and naked singularities in astrophysical phenomena (see Kovacs \& Harko 2010; Pugliese, Quevedo \& Ruffini 2011). 
5. Finally, further considerations of quantum gravity effects in the vicinity of naked singularities, which are super-ultra-strong gravity regions, could yield intriguing theoretical insights into the phenomenon of gravitational collapse (Goswami, Joshi \& Singh 2006).

\section{Acknowledgments}

Over past years, discussions with many colleagues and friends have contributed greatly to shape my understanding of the questions discussed here. In particular, I would like to thank I. H. Dwivedi, N. Dadhich, R. Goswami, T. Harada, S. Jhingan, R. Maartens, K. Nakao, R. Saraykar, T. P. Singh, R. Tavakol, and also many other friends with whom I have extensively discussed these issues. Fig. 4 here is from Mena, Tavakol \& Joshi (2000), and Fig. 6 is from Patil, Joshi \& Malafarina (2011). The rest of the figures are from Joshi (2008).

\section{References}

Baiotti L., Rezzolla L., 2006, Phys. Rev. Lett., 97, 141101

Banados M., Silk J., West S.M., 2009, Phys. Rev. Lett., 103, 111102

Barausse E., Cardoso V., Khanna G., 2010, Phys. Rev. Lett., 105, 261102

Berti E., Cardoso V., Gualtieri L., Pretorius F., 2009, Phys. Rev. Lett., 103, 239001

Chandrasekhar S., 1934, Observatory, 57, 373

Datt B., 1938, Z. Physik, 108, 314

Goswami R., Joshi P.S., 2007, Phys. Rev. D, 76, 084026

Goswami R., Joshi P.S., Singh P., 2006, Phys. Rev. Lett., 96, 031302

Grib A.A., Pavlov Y. V., 2010, arXiv:1004.0913 [gr-qc].

Hawking S. W., Ellis G.F.R., 1973, The Large Scale Structure of Space-time, Cambridge University Press, Cambridge

Harada T., Iguchi H., Nakao K., 2000, Phys. Rev. D, 61, 101502

Harada T., Iguchi H., Nakao K., 2002, Prog. Theor. Phys., 107, 449

Harada T., Iguchi H., Nakao K., Singh T.P., Tanaka T, Vaz C., 2001, Phys. Rev. D, 64, 041501

Hod S., 2008, Phys. Rev. Lett., 100, 121101

Hubeny V.E., 1999, Phys. Rev. D, 59, 064013

Jacobson T., Sotiriou T.P., 2009, Phys. Rev. Lett., 103, 141101

Jacobson T., Sotiriou T.P., 2010a, J. Phys. Conf. Ser., 222, 012041

Jacobson T., Sotiriou T.P., 2010b, Phys. Rev. Lett., 104, 021101

Joshi P.S., 2008, Gravitational Collapse and Spacetime Singularities, Cambridge University Press, Cambridge.

Joshi P.S., Dwivedi I.H., 1999, Class. Quantum Grav., 16, 41

Joshi P.S., Krolak A., 1996, Class. Quant. Grav., 13, 3069

Joshi P.S., Malafarina D., 2011, Phys. Rev. D, 83, 024009

Joshi P.S., Dadhich N., Maartens R., 2002, Phys. Rev. D, 65, 101501

Kovacs Z., Harko T., 2010, Phys. Rev. D, 82, 124047

Matsas G. E.A., da Silva A.A.R., 2007, Phys. Rev. Lett., 99, 181301

Matsas G.E.A., Richartz M., Saa A., da Silva A.A.R., Vanzella D.A.T., 2009, Phys. Rev. D, 79, 101502

Mena F.C., Tavakol R., Joshi P.S., 2000, Phys. Rev. D, 62, 044001

Oppenheimer J.R., Snyder H., 1939, Phys. Rev., 56, 455. 
Patil M., Joshi P.S., 2010, Phys. Rev. D, 82, 104049

Patil M., Joshi P.S., 2011a, arXiv:1103.1082 [gr-qc]

Patil M., Joshi P.S., 2011b, arXiv:1103.1083 [gr-qc]

Patil M., Joshi P.S., Malafarina D., 2011, Phys. Rev. D., 83, 064007

Penrose R., 1969, Riv. Nuovo Cimento Soc. Ital. Fis., 1, 252

Pugliese D., Quevedo H., Ruffini R., 2011, arXiv:1103.1807 [gr-qc]

Richartz, M., Saa A., 2008, Phys. Rev. D, 78, 081503

Report of the Physics Survey Committee 1986, Physics through the 1990s: gravitation, cosmology, and cosmic-ray physics, National Academy Press, Washington, D.C.

Wald R.M., 1997, gr-qc/9710068

Wei S.-W., Liu Y.-X., Heng G., Fu C.-E., 2010, Phys. Rev. D, 82, 103005

Zaslavskii O.B., 2010, JETP Lett., 92, 571 